\title{
De la sécurité dans les milieux universitaires : cas du campus de l'université de Lubumbashi
}

\author{
Par MWANZA WA KALOMBO Claude* et MUMBA KAKUDJI Martial**
}

\section{INTRODUCTION}

La sécurité peut se définir comme un état d'esprit confiant et tranquille qui résulte du sentiment, bien ou mal fondé, que l'on est à l'abri de tout danger. Elle peut aussi être considérée comme une situation objective, reposant sur des conditions matérielles, économiques, politiques, qui entraine l'absence de danger pour les personnes ou de menaces pour les biens et qui détermine la confiance.

Pour P.F. Brubière, la sécurtié est l'état (c'est-à-dire situation ou état de situations) correspondant à l'absence de péril ou à la très grande improbabilité de péril. L'expression être en sécurité, dit-il, ce n'est pas avoir le péril à redouter ou n'avoir qu'un péril très faible à craindre. Autrement-dit, même si tout est réglementé sur les cités universitaires, une situation d'insécurité peut toujours avoir lieu. Et donc, il serait aberrant de ne plus l'imaginer' ${ }^{1}$.

Actuellement, le monde vit une situation d'insécurité de tout genre. Dans la ville de Lubumbashi, il ne se passe pas deux jours sans que sa population n'assiste ou n'apprenne par la voie des ondes que dans tel ou tel autre quartier les actes d'insécurité y ont été perpétrés. Etant donné que la société subit différentes formes de violences, les milieux universitaires en République Démocratique du Congo et particulièrement celui de l'Université de Lubumbashi n'en sont pas épargnés, il en est de même pour les milieux scolaires, du travail, et autres.

La violence est une action brutale, physique ou morale envers quelqu'un. Elle consiste à agir sur quelqu'un ou à le forcer contre sa volonté en utilisant la force physique ou psychique.

En cas d'espèce, les milieux universitaires sont de lieux en principe où doivent régner le calme mais de fois, ils s'affichent comme de milieux d'insécurité surtout quand les étudiants s'engagent à faire des réclamations relatives à leurs besoins. Ils recourent à l'usage de moyens non appropriés sous prétexte qu'ils revendiquent leurs droits aux fins de satisfaire leurs besoins. Il s'agit aussi d'un endroit qui réunit les personnes provenant de différentes familles, religions, cultures, etc. Au choc de plusieurs familles, cultures et religions, s'enchevêtrent autant de comportements et mentalités à gérer sur les cités universitaires.

* Assistante à la Faculté de Droit de l'Université de Lubumbashi.

** Chef de Ttravaux à la Faculté de Droit de l'Université de Lubumbashi, DEA en Droit Public et Avocat au Barreau de Lubumbashi.

1 Katambwe Malipo Gérard, Syllabus de Droit de la sécurité internationale, UNILU, L2 Droit, Département de Droit public, 2005-2006, inédit. 
Dans pareille occurrence, l'insécurité serait aussi inévitable. Elle est, peut-être, dans les esprits sans être manifestés au départ mais par la suite, elle peut toujours s'éclore et ce, à tout moment.

Bien qu'ils peuvent être illustratifs, quelques cas qui peuvent conduire à l'insécurité sur les cités universitaires au quotidien dans nos milieux universitaires et causent de protestations sont les suivants : l'interruption de la fourniture d'eau et d'électricité; les agressions ou tracasseries des Policiers aux alentours de cités universitaires du Campus de Lubumbashi; l'arrestation d'un voleur au campus par les étudiants; le paiement frais académiques que les étudiants jugent exorbitants etc...

Ces situations peuvent d'une manière ou d'une autre motiver l'insécurité dans les milieux universitaires. Ce qui fait que la question de la sécurité ou de la violence dans les milieux universitaires devienne récurrente.

Pour bien analyser la situation sécuritaire dans les milieux universitaires, il sied de répondre à une série des questions à savoir :- Quelles sont les causes d'insécurité et quels en sont les auteurs dans les milieux universitaires à Lubumbashi? (A) - Quelles sont les mesures et services de sécurité dans ces milieux? (B) et Quelles peuvent être les solutions? (C).

Telles sont les préoccupations auxquelles nous tenterons de répondre tout au long de cette rédaction.

\section{A. Les causes de la violence ou insécurité}

Comme nous venons de le dire dans les milieux universitaires la violence est de tout genre. De manière générale, on peut citer : le vol; les injures; les menaces; les agressions verbales ou physiques; le harcèlement sexuel ou moral; viol; l'interruption de la fourniture d'eau et d'électricité; les agressions ou tracasseries des Policiers aux alentours de cités universitaires du Campus de Lubumbashi; l'arrestation d'un voleur au campus par les étudiants; le paiement frais académiques que les étudiants jugent exorbitants etc...

Ces actes constituent un frein à l'évolution académique des étudiants d'une part et du personnel administratif, scientifique et académique d'autre part.

Alors quelles en sont les causes? Il sied de noter que les causes de ces situations sont multiples. Et nous les classifions selon qu'elles peuvent être liées au fonctionnement et à l'insuffisance des infrastructures (I) ou liées à la conjoncture sociale, économique et politique (II).

\section{Les causes liées au fonctionnement de l'université et l'insuffisance des infrastructures.}

Il est vrai que le fonctionnement d'une institution, selon qu'il peut être bon ou mauvais, peut être considéré comme une cause de tentions, violences et insécurité d'une part et aussi peut jouer le rôle de garantir la sécurité et la tranquillité d'autre part. 
L'on peut estimer que du point de vue du fonctionnement de l'Université de Lubumbashi, les points ci-après peuvent être les causes d'insécurité dans les milieux universitaires.

Il s'agit de :

1. La capacité d'accueil de nos universités est largement dépassée

La capacité d'accueil et logement des étudiants est devenue inférieure au nombre d'étudiants qui doivent être logés. Pour s'en rendre compte, la construction des homes de l'Université de Lubumbashi a été faite pour deux étudiants par chambre, de ce fait, les placards, les chaises, les lits ne sont que deux par chambre. Hélas, à ce jour, seuls le home 10 (pour les garçons), home 2 et 6 (pour les filles) répondent à ces critères. Les restent des homes et blocs ne répondent pas aux critères d'un bon logement. Les étudiants y sont installés à quatre part chambre. De ceci, on peut évoquer notamment, l'insécurité sanitaire suite aux mauvaises conditions d'hébergement.

Cette situation peut générer l'insécurité vis-à-vis des étudiants, dans la mesure où les étudiants sont dans l'obligation de rester deux sur un lit d'en haut et deux sur le lit d'en bas, et leurs biens pour deux étudiants doivent être conservés dans un même placard, ce qui a pour conséquence, si un étudiant partage le placard avec un voleur, il sera victime de vol.

Un autre problème se pose dans la mesure où les portes de certaines chambres ne sont plus en bon état, et comme la plupart des homes, les portent principales d'entrée sont ouvertes, la nuit les voleurs (internes tout comme externes) peuvent venir voler les biens des étudiants en pleine nuit.

2. Pas suffisamment d'auditoires, leur délabrement, manque ou insuffisance des sièges, de matériel de sonorisation (micro, amplificateurs, les haut-parleurs etc.)

L'insécurité peut aussi avoir pour cause l'insuffisance d'auditoires parce que les étudiants sont entassés dans un auditoire avec les sièges insuffisants, ce qui crée une certaine promiscuité. Cette situation génère l'insécurité dans les occurrences ci-après :

- lors qu'il fait chaud, les étudiantes tombent de fois en plein enseignement;

- les voleurs peuvent s'en servir de cette promiscuité pour déplacer facilement les biens de leurs voisin(e)s;

- les étudiants peuvent se disputer les places (sièges) et cela peut aboutir aux affrontements violents, menaces et même les insultes.

3. Les installations sanitaires insuffisantes, en mauvais état et mal entretenues

L'insécurité peut aussi résulter d'insuffisance des installations sanitaires et de manque d'entretien de ces infrastructures sanitaires existantes par les étudiants eux-mêmes à qui revient 
ce devoir et en même cette obligation. Lors que ces installations sont bouchées ${ }^{2}$, les étudiants souffrent parce qu'ils ont difficile à trouver des endroits où ils peuvent aller faire leurs besoins, et aussi cela peut provoquer certaines maladies d'insalubrité. C'est ainsi qu'il y a lieu de considérer que c'est une autre sorte d'insécurité à laquelle les étudiants sont exposés dans les milieux universitaires.

\section{L'insuffisance des homes et le surnombre d'étudiants dans les chambres}

Les milieux universitaires sont inondés de plusieurs étudiants provenant de partout lorsqu'on s'imagine l'immensité du territoire national de la République Démocratique du Congo. Tous ne suffisent pas dans les homes disponibles, par exemple à l'Université de Lubumbashi. Parce qu'ils manquent d'endroit où aller logés, les étudiants sollicitent malgré ce fait l'hébergement dans le campus tout en acceptant ces conditions de vie précaires. Le surnombre est visible dans les homes.

5. Les mauvaises conditions de vie et de travail des enseignants et du personnel universitaire

Les mauvaises conditions de vie et de travail des enseignements et du personnel universitaire peuvent aussi en quelque sorte déboucher à une certaine insécurité financière vis-à-vis des étudiants. Cela peut s'expliquer de cette manière : les enseignants, nul l'ignore, ne sont toujours pas bien payés pour la plupart. Certains cherchent à vendre les supports en vue de pallier aux charges familiales nombreuses. Face à cette situation, les étudiants peuvent recourir à plusieurs moyens même illégaux afin de se procurer de supports. C'est dans cette occurrence que certains volent les ordinateurs, téléphones et d'autres biens de valeurs de leurs voisins.

\section{La maque de fourniture en eau potable et électricité}

D'ores et déjà fixons que c'est cette situation qui a été à la base des tristes évènements de février de l'an 2019 à l'Université de l'Université.

L'électricité dans les milieux universitaires permet aux étudiants de préparer, de repasser leurs habits, de lire, de travailler avec leurs machines (ordinateurs) pour les travaux pratiques, les travaux dirigés, les travaux de fin de cycle et mémoires. Grâce à l'électricité, les équipes de sécurité du campus, pendant la nuit, arrivent à lutter tant soit peu, contre l'insécurité. L'électricité, dit-on, a été proposée par les criminologues comme un substitut pénal dans la mesure où elle lutte contre la criminalité ou l'insécurité.

L'eau aussi joue un rôle très important dans la vie quotidienne et surtout dans les milieux universitaires comme au campus de Lubumbashi car pour lessiver, se laver, préparer,

2 On note que ces installations pour un plus grand nombre sont déjà dans un mauvais été demandant la réhabilitation d'autant plus qu'elles se bouchent fréquemment. 
faire les différents besoins, l'on a besoin de l'eau. Alors, le maque de fourniture en eau et électricité peut être considérée comme une cause de l'insécurité d'autant plus que les étudiants sans électricité et eau ne trainent pas de manifester et faire de grognes. Pendant ces manifestations, les actes de violence, vol, injures et autres peuvent être posés. Notons le fait que, lors de manifestations des étudiants, ces derniers ne veulent pas voir la Police (alors que celle-ci est le premier service de sécurité et de sauvegarde de l'ordre public). Si elle intervient, c'est là que les émeutes, les violences peuvent commencer au point de se généraliser. Et ainsi, elles peuvent déborder et créer de la panique dans la ville et dans le chef de la population.

\section{Les causes liées à la conjoncture sociale, économique et politique}

La situation sociale, économique et politique est aussi l'une des causes de l'insécurité dans les milieux universitaires.

\section{La cause économique d'insécurité}

Vu que la situation économique du pays s'est réellement dégradée, beaucoup d'étudiants vivant dans la précarité, éprouvent de réelles difficultés à s'acquitter des différents frais académiques; à se procurer les supports pour les enseignements dispensés; à consulter les bibliothèques; à payer les moyens de transport pour atteindre les cités universitaires. Bref, satisfaire les besoins fondamentaux scientifiques et académiques.

Cependant, cette situation débouche parfois aux actes de vols, escroqueries, extorsion, abus de confiances et d'autres actes similaires pouvant être constatés à plusieurs niveaux.

\section{La cause socio-culturelle}

Les milieux universitaires réunissent les personnes de différentes cultures et croyances. Certains viennent avec le seul but d'étudier, trouver les diplômes et se lancer dans la société. Mais pour certains autres, ce n'est pas le cas. Ils viennent pour voler, pour la prostitution, pour les bisness etc.

Et certains autres encore viennent dans les auditoires sans même que leurs noms ne soient connus par l'Administration de l'Université. Ce sont les personnes se trouvant dans cette deuxième catégorie qui sont souvent et surtout à la base des actes de violences et d'insécurité dans les cités universitaires de l'Université de Lubumbashi.

A cet effet et par manque des repères, le laxisme des familles et le complexe des étudiants qui se croient arrivés, veulent s'affirmer en contestant l'autorité et l'ordre établi; s'affichant par la tenue vestimentaire, la coiffure... Il en est de même de la provenance de milieux sociaux différents (c'est-à-dire le fait d'avoir les nationalités, éducations, coutumes 
et mœurs différentes ${ }^{3}$ ). Tout ceci peut être générateur d'une autre forme d'insécurité dans les milieux universitaires.

\section{La cause politique}

Nonobstant le caractère apolitique des milieux universitaires, ils deviennent de plus en plus politisés. Cette politisation est souvent à la base de l'insécurité. Elle est même motivée par certains étudiants qui courent derrière les politiques pour avoir de l'argent tout en leur rassurant qu'ils peuvent y trouver un électorat facile à conquérir par différentes manœuvres. Souvent, ce sont des étudiants d'une même obédience qui traite en coulisses avec les leurs et qui influencent les autres qui se laissent prendre pour avoir de l'argent afin de subvenir à leurs besoins. Et pourtant, la politique est interdite dans ces milieux.

Les milieux universitaires regorgent pour le moment des enseignants et étudiants faisant partie de tel ou tel parti politique. Ceci est à la base d'un certain clivage et peut susciter des tensions. De la sorte, la paix risque de ne pas être retrouvée en ce moment-là. Plusieurs soulèvements enregistrés au campus de Lubumbashi seraient dus à la politisation du secteur dans les coulisses de certains hommes politiques.

Les étudiants comme nous venons de le voir dans le point consacré à la cause économique, n'ayant pas de moyens nécessaires pour satisfaire leurs besoins, se trouvent être faciles à manipuler et à utiliser par les politiques à des fins multiples, parmi lesquelles le sabotage des institutions de l'Etat et les autorités académiques.

\section{B. Les mesures et les services de sécurité}

Peut-on dire qu'aucune mesure n'est prise pour lutter contre cette insécurité?

Il existe déjà des mécanismes pour contrer cette violence et assurer la sécurité à l'Université de Lubumbashi. A côté des mesures prévues, il existe aussi les services appelés à mettre en œuvre ces mesures.

A l'Université de Lubumbashi, c'est le Recteur qui est le garent de la sécurité. Il est secondé dans sa tâche par le Secrétaire général académique (I) et Secrétaire général administratif (II).

3 Pour certaines cultures le vol peut être considéré comme une valeur, un garçon qui n'a jamais été arrêté avec une fille est considéré comme un malade sexuel. Un tel garçon lors qu'il arrive dans les cités universitaires, avec sa culture, il peut voler comme bon lui semble, violer les filles d'autrui dans les endroits sombres. 


\section{Le Secrétaire général administratif}

Le Secrétaire général administratif reçoit les rapports de sécurité de :

$1^{\circ}$ le Conseiller juridique;

$2^{\circ}$ la Direction des œuvres estudiantines;

$3^{\circ}$ la Police universitaire, la Mairie générale et la Délégation générale des étudiants.

\section{Le rôle du Conseiller juridique}

Le Conseiller juridique en vertu de ses attributions, il coordonne et régule dans les limites de ses compétences la sécurité de l'Université, ses biens et toutes les personnes qui se trouvent dans les milieux universitaires. Il veuille aussi au respect des lois et règlements qui régissent l'Université tant par le personnel que par les étudiants. Ce dernier est assisté dans l'exercice de ses attributions par un adjoint et plusieurs attachés juridiques. Il travaille et collabore avec les services de sécurité dont la Police ou garde universitaire et la Mairie générale. La sécurité est sous la charge du Conseiller juridique au cabinet de Monsieur le Recteur.

La direction des œuvres estudiantines est dirigée par le Directeur des œuvres qui, en vertu de ses attributions, il veuille à la sécurité dans les cités universitaires. Il joue un rôle aussi important dans le maintien de l'ordre dans les milieux universitaires d'autant plus que c'est lui qui loge les étudiants et qui peut, par ses stratégies de travail, identifier les acteurs d'insécurité dans ces milieux.

\section{Le rôle du Directeur des œuvres}

Le Directeur des œuvres aussi travaille en collaboration avec la Police universitaire qui est chargée de la sécurité et s'appuie sur la Mairie générale et son équipe et le Délégué général des étudiants. Il y a lieu de constater que la Police universitaire est composée d'Officiers de Police judiciaire assermentés. Cependant, ils sont de fois dépourvus de moyens matériels pouvant permettre leur mobilité et leur efficacité. D'où la nécessité de pourvoir des différents moyens pour la réalisation de sa politique. Leur nombre est insuffisant. Il est mieux qu'elle soit renforcée.

3. Le rôle de la Police universitaire, de la Mairie générale et de la Délégation générale des étudiants

La police universitaire joue un rôle non négligeable dans la sécurisation des milieux universitaires. Elle arrête les semeurs de troubles, elle procédure par ses Officiers de la police judiciaire assermentés aux enquêtes et aux auditions pour dénicher la vérité. Après instruction des dossiers par eux, ils les transmettent au Conseiller Juridique pour que ce dernier propose de sanctions à prendre par le Recteur et ce, conformément aux règlements de l'Univer- 
sité. Mais au cas où les faits s'avéraient graves parce que violant les lois de la République, les dossiers seront transférer auprès des instances judiciaires pour que le droit y soit dit.

La Mairie est composée de quatre vices Maires généraux. Chacun a ses attributions. Il y a celui qui est chargé de l'administration; de la sécurité; du social et enfin, de la salubrité et l'environnement.

Ils sont recrutés parmi les étudiants pour assurer la sécurité. Ils sont désignés sur base d'un certain nombre des critères objectifs. Ils participent activement à la sécurisation des cités universitaires. Ils sont désignés pour cette fin. Mais certains par manque de formation confondent leur rôle en croyant qu'ils sont devenus les touts puissants et intouchables. Ainsi, ils deviennent eux même à l'origine de violences, causant ainsi l'insécurité.

Cependant, leur présence est tolérée des étudiants parce qu'il s'agit de leurs pairs. Il serait souhaitable qu'ils soient formés et choisis en fonction de leur exemplarité.

La Délégation générale a pour rôle de représenter les étudiants au Conseil de l'Université présidé par le Recteur ${ }^{4}$.Le Délégué général travaille en collaboration avec le Maire général. Il préside le Conseil consultatif et le présidium. Il assiste les étudiants en difficulté devant les autorités. Il fait divulguer le règlement de l'Université et des étudiants pour que ces derniers l'observent strictement. Il doit divulguer les valeurs afin que chaque étudiant ait à cœur de veiller particulièrement à la bonne réputation de l'Etablissement. L'étudiant doit en toute circonstance, obéissance et respect à l'autorité académique et à tout membre du personnel de l'Etablissement qui la représente ${ }^{5}$.

\section{Le Secrétaire général académique}

Le Secrétaire général académique obtient des doyens les rapports de sécurité des différentes facultés c'est-à-dire le déroulement des activités académique et scientifiques.

L'exécution et la réalisation des charges horaires.

La situation des enseignants (maladies,)

Cette sécurité s'organise sur base de différents textes, à savoir le vadémécum du gestionnaire, le règlement organique de l'université, le statut de l'université et de la fonction enseignante, le guide de l'étudiant, les arrêtés du Ministre de l'enseignement supérieur et universitaire.

Ces différents services sécuritaires travaillent en collaboration pour que les objectifs de l'Université soient réalisés à savoir : l'enseignement, la recherche et les services à la société.

4 Règlement organique de l'Université de Lubumbashi, Lubumbashi, mise à jour 2005-2006, p.1.

5 Vade-mecum du gestionnaire d'une institution d'enseignement supérieur et universitaire, $3^{\text {ème }}$ édition, Kinshasa, octobre 2014, p.85. 


\section{Perspectives ou solutions}

Il est difficile de faire disparaitre complètement l'insécurité ou violence dans les cités universitaires, car elle fait partie de toute société humaine. Cependant, il est possible de la diminuer sensiblement.

C'est pourquoi il faut principalement s'attaquer à ses causes.

1. L'augmentation des infrastructures universitaires ou leur décentralisation.

Il faut créer de nouveaux espaces universitaires qui tiennent compte de nombre croissant d'étudiants et de structures adéquates (sanitaires, bureaux, homes, réfectoires). C'est dans cette perspective que l'Université de Lubumbashi par le biais de son Recteur a acquis un espace dénommé UNILU II (Université de Lubumbashi II).

2. L'éveil des étudiants à des activités intellectuelles, améliorer la recherche.

3. Former les étudiants à la culture de la paix, la citoyenneté, la transmission des valeurs.

4. Délimiter ou clôturer l'espace universitaire pour éviter des intrusions intempestives.

5. Il faut une volonté politique réelle des autorités politique et administratives d'améliorer les conditions socio-économiques de la population en général et les conditions de travail des enseignants c'est-à-dire du personnel académique et scientifique et du personnel administratif.

6. L'implication des familles, surtout des parents dans le suivi de leurs enfants, élevés comme étudiants.

7. L'implication des enseignants dès le niveau primaire et secondaire pour une meilleure éducation et instruction des enfants.

8. La collaboration des différents services de sécurité, le renouvellement des effectifs et leur formation et leur dotation en moyens matériels adéquats.

9. L'implication de tous à tous les niveaux pour lutter contre la violence au sein de notre société en général.

\section{CONCLUSION}

En conclusion, il convient de noter que les milieux universitaires sont de lieux en principe où doivent régner le calme mais de fois, ils s'affichent comme de milieux d'insécurité surtout quand les étudiants s'engagent à faire des réclamations relatives à leurs besoins. Ils recourent à l'usage de moyens non appropriés sous prétexte qu'ils revendiquent leurs droits aux fins de satisfaire leurs besoins. Il s'agit aussi d'un endroit qui réunit les personnes provenant de différentes familles, religions, cultures, etc. Au choc de plusieurs familles, cultures et religions, s'enchevêtrent autant de comportements et mentalités à gérer sur les cités universitaires. Dans pareille occurrence, l'insécurité serait aussi inévitable. Elle est, peut-être, dans les esprits sans être manifestés au départ mais par la suite, elle peut toujours s'éclore et ce, à tout moment. Pour 
Pour remédier à l'insécurité dans les milieux universitaires, les pistes des solutions dans le développement de cet article peuvent servir des mesures de sécurité. La sécurité c'est la vigilance, la prévention et la protection de tous par tous. 